\title{
Micromorphology of Some Sclerotial Actinomycetes and Development of Their Sclerotia
}

\author{
By P. L. GANJU AND M. R. S. IYENGAR \\ Microbiological Research and Development Laboratories, \\ Alembic Chemical Works Co. Ltd, Baroda, India
}

(Received I4 May 1973; revised 30 November 1973)

\begin{abstract}
SUMMARY
Microscopic studies on four Chainia species have revealed that their vegetative mycelia were characterized by a rapid sclerotization resulting in the formation of broad, closely septate sclerotial hyphae measuring I to $3 \mu \mathrm{m}$ in width. The sclerotia which subsequently formed originated from well-defined sclerotial initials which developed further by growth and division in different planes. A study of the internal structure of the sclerotia by microtomy revealed that they possessed a typical pseudoparenchymatous structure without any internal differentiation into zones. The sclerotia developed in different ways. In Chainia barodensis and $C$. poonensis, the sclerotial initials were intercalary. In $C$. ochracea they arose laterally on multihyphal strands as is characteristic of many fungi. In Chainia 33 br the sclerotial initials were both intercalary and lateral. In $C$. poonensis, $C$. ochracea and Chainia $33 \mathrm{br}$ the substrate growth sometimes formed a stroma-like crust with sclerotia anchored to or embedded in it. Scanning electron microscopy of sclerotia of $C$. barodensis showed that these structures possessed a characteristic surface architecture. The actinomycete sclerotium is not a simple hyphal aggregation but a definitive morphological entity with characteristic modes of development.
\end{abstract}

\section{INTRODUCTION}

Sclerotia, which are discrete vegetative structures of multihyphal origin with varying degrees of differentiation, have long been known to be resistant bodies characteristic of fungi (see review by Willetts, 197I). Knowledge concerning the development and anatomy of these structures has been reviewed (Butler, 1966; Willetts, 1972). In contrast, the presence of sclerotia among actinomycetes did not attract attention until Thirumalachar (I955) described the genus Chainia based on its ability to produce such bodies. Many more sclerotial actinomycetes have since been described (Kalakutskii \& Krassilnikov, 1960; Kuznetsov, 1962; Thirumalachar \& Sukapure, I964; Thirumalachar, Rahalkar, Deshmukh \& Sukapure, 1965; Thirumalachar, Sukapure, Rahalkar \& Gopalkrishnan, 1966), but information regarding the nature of the sclerotial bodies produced by them is still scanty (Baldacci, Locci \& Locci, 1966). We have further studied the morphology and the development of the actinomycete sclerotium.

\section{METHODS}

Micro-organisms. The following cultures were used: Chainia barodensis (P. L. Ganju and M. R. S. Iyengar, unpublished), C. poonensis and C. ochracea (Kuznetsov, 1962; Shirling \& Gottlieb, 1972), a new isolate of Chainia designated Chainia 33br (unpublished) and Streptomyces griseus ATCCIOI37. Mycelial suspensions of monocolony isolates of these cultures obtained from aerated submerged growth were frozen at about $-70^{\circ} \mathrm{C}$ and used as stocks. The cultures were viable even after 2 years of storage. 
Media. Glycerol asparagine agar (GAA), yeast extract agar (YEA) (Shirling \& Gottlieb, 1966), oat meal agar (Waksman, I96I) supplemented with I \% glucose (OMA), and yeast dextrose agar (YDA) (Waksman, 196I) were used.

Preparation of coverslip cultures for microscopic examination. The cultures were grown on GAA and OMA on coverslips at $25^{\circ} \mathrm{C}$ by means of the agar-cylinder technique of Nishimura \& Tawara (1957). Such cultures were fixed in Chabaud's mixture (Tulasne \& Vendrely, 1947), washed with water, air-dried, and stained when necessary.

Microtomy. Cultures were spot-inoculated on GAA or YEA plates and allowed to grow for 2 to 3 weeks at $25^{\circ} \mathrm{C}$. Small rectangular blocks of growth were excised from the plates and fixed in formalin-aceto-alcohol, dehydrated and embedded in paraffin wax, according to the procedure given by Purvis, Collier \& Walls (1964). The cultures were cut tangentially on a rotary microtome and the I to $2 \mu \mathrm{m}$ thick sections were mounted on slides by means of egg albumin, dried and finally deparaffinized with chloroform.

Staining. Coverslip cultures were stained by using a modification of Robinow's membranestaining technique (Klieneberger-Nobel, 1947). The cultures were mordanted with a $5 \%$ (w/v) aqueous tannic acid solution for $30 \mathrm{~min}$, washed with water and stained with $0.0 \mathrm{I} \%$ aqueous crystal violet for $3 \mathrm{~min}$.

Some microtome sections were stained with 0.5 to $\mathrm{I} \cdot 0 \%$ aqueous safranin.

Microscopy and photomicrography. A Carl Ziess Universal research microscope fitted with Neofluar bright-field or phase objectives and an achromatic-aplanatic condenser was used for observing the cultures. Orwo or Kodak panchromatic film, 100-125 ASA, was used in photomicrography. Unstained preparations were mounted in paraffin oil and examined under phase contrast. The stained preparations were observed either directly under immersion oil or after mounting in paraffin oil.

Scanning electron microscopy (s.e.m.). A JSM-U ${ }^{3}$ scanning electron microscope (JEOL Ltd, Tokyo, Japan) was used to observe the surface structure of the whole sclerotia of Chainia barodensis and the surface growth of $C$. ochracea. Sclerotia of $C$. barodensis were obtained from two-week-old GAA slants by scraping. They were fixed in Chabaud's mixture, washed and dehydrated. Small pieces of surface growth were also excised from osmic acidfixed two-week-old YDA cultures of $C$. ochracea and dehydrated. The dehydrated materials were coated with carbon and gold and examined by s.e.m. The general procedures employed in s.e.m. were those of Williams \& Davies (1967).

\section{RESULTS}

Micromorphology of early mycelial growth

Overnight coverslip cultures of all the actinomycetes under study revealed only simple multicellular radiating hyphae. After longer periods of growth, Chainia barodensis, $C$. poonensis, C. ochracea and Chainia 33 br showed extensive sclerotial activity; hyphae with broad sclerotial cells could be seen prominently against the background of normal hyphae (Fig. I). Comparisons between these sclerotial actinomycetes and Streptomyces griseus ATCCIOI37 clearly indicated that in the former there was a gradual transition from the normal hyphae, which were generally less than I $\mu \mathrm{m}$ wide, to the thicker sclerotial ones measuring I to $2 \mu \mathrm{m}$ or sometimes up to $3.5 \mu \mathrm{m}$ as in C. barodensis and C. poonensis. S. griseus did not show any such differentiation and its hyphae were never more than I $\mu \mathrm{m}$ in diameter. No hyphal aggregations which had even a remote resemblance to the sclerotia formed by Chainia species could be discovered in this organism at any stage of its growth.

Many important characteristic features were observed in the sclerotial cultures from the 

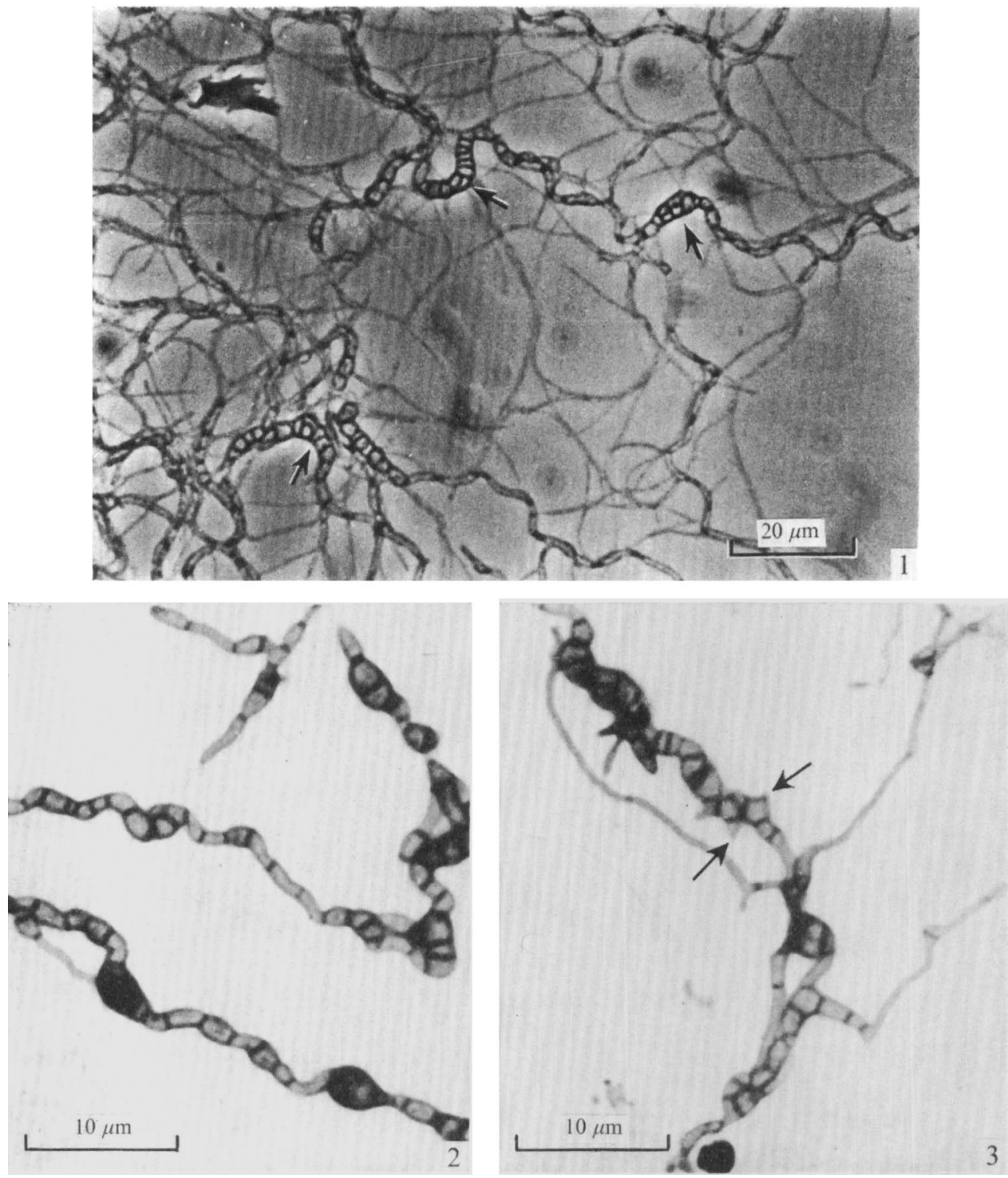

Fig. I. Chainia barodensis. Mycelial growth showing broad, closely septated, sclerotial hyphae with some of the cells dividing by septa perpendicular to the main transverse walls (arrows); OMA, phase contrast.

Fig. 2. Chainia poonensis. Multicellular hyphae with broad intercalary sclerotial cells; GAA, tannic acid-crystal violet.

Fig. 3. Chainia poonensis. Sclerotial cells with profuse septation; branch initials are also seen (arrows); GAA, tannic acid-crystal violet.

early stages of their growth. The sclerotial hyphae of these cultures generally showed prominent walls and cross-septa. In Chainia barodensis, $C$. poonensis and Chainia 33br, the septa were laid down profusely, sometimes so close to each other that the resulting structures appeared discoid. Another feature of the sclerotial cultures was the occasional septation in different planes. This was more readily demonstrated in C. barodensis than in C. poonensis and Chainia 33br, and was never observed in C. ochracea. 

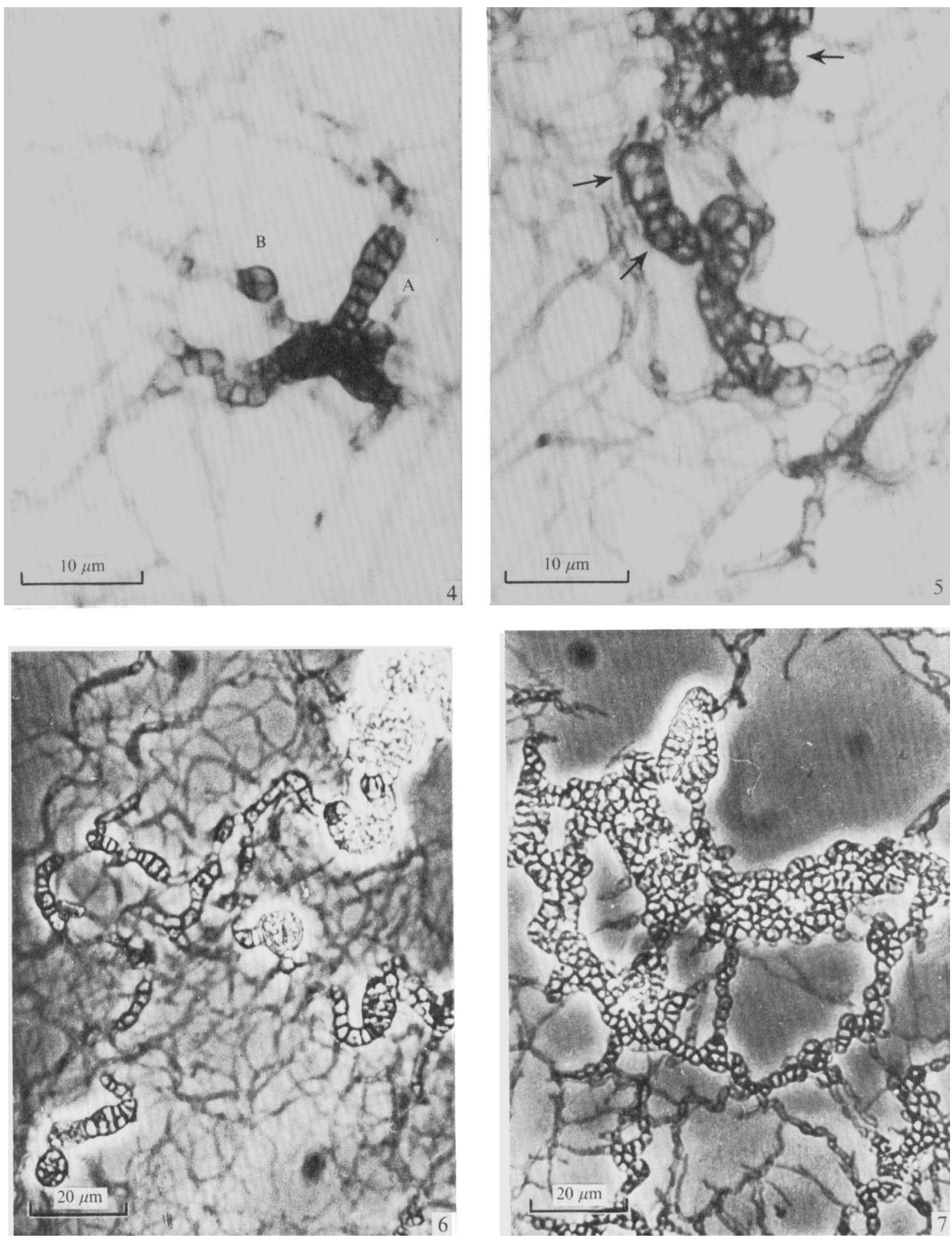


\section{Ontogeny of sclerotia}

Chainia barodensis, C. poonensis and Chainia $33 \mathrm{br}$ exhibited more or less similar characteristics in their sclerotial development. More detailed studies were carried out with C. barodensis. In C. barodensis, C. poonensis and Chainia $33 \mathrm{br}$, long sections of the multicellular hyphae showed a strong tendency to segment. At several points in these hyphae small localized areas enlarged conspicuously and became delimited by new septa, resulting in the formation of more or less ellipsoid sclerotial cells. Segmentation of many adjacent lengths of the hyphae resulted in their transformation into chains of sclerotial cells - the sclerotial hyphae (Figs. 2 and 3 ). This process appeared to be more vigorous in Chainia poonensis and Chainia $33 \mathrm{br}$ than in $C$. barodensis, thus transforming major portions of the parent hyphae of $C$. poonensis and Chainia 33 br into broad sclerotial filaments. In $C$. poonensis, a feature of the younger hyphae was the delimitation of solitary, round-toellipsoidal, bead-like segments at more distant places and the extension of further sclerotial activity from these centres (Figs. 2 and 3). In all the cultures the sclerotial hyphae also branched but the branching was not as profuse as in the usual hyphae. During branching a sclerotial cell protruded at a point and gave rise to a spindle-shaped out-growth (Fig. 3). The outgrowth then enlarged in size, delimited itself from the mother cell and continued to divide linearly giving rise to new sclerotial hyphae.

Frequently, certain cells in the sclerotial hyphae, which we have called the sclerotiumforming initials, further enlarged in size and were divided by septa laid in different planes (Fig. I). The developmental stages leading to the formation of small sclerotial bodies in Chainia barodensis are presented in Figs. 4 and 5.

Later stages of development of sclerotia were difficult to observe in the coverslip cultures as the structures became more sclerotic and refractive to staining. The detailed structure of large, unstained sclerotial bodies could not be clearly observed by phase-contrast microscopy because of extensive light scattering (Figs. 6 and 7). However, by repeated and careful observation of the coverslip cultures of Chainia barodensis, it could be inferred that these structures were formed by a further enlargement, branching and septation of the earlier stages. Sclerotia were generally discrete (Fig. 6) but synchronous and active multiplication of several adjacent cells in a hypha could lead to the formation of irregular compound sclerotia. In Chainia 33br (Fig. 7) and C. poonensis, discrete sclerotia were not formed in the coverslip cultures and flat masses of sclerotial cells were observed.

The hyphae of Chainia $33 \mathrm{br}$ often showed a strong tendency to run in close proximity, wall to wall, without any demarcation between the abutting walls (Fig. 8), forming hyphal aggregations which resembled, in miniature, the hyphal strands formed in some fungi. Sclerotial buds were seen to arise laterally on these multicellular hyphae which then divided further to form the sclerotial filaments.

Fig. 4. Chainia barodensis. A short thick sclerotial hypha with many of its cells dividing by septa at right-angles to the transverse walls (A); a solitary cell with cross-walls in two planes is also seen (B); GAA, tannic acid-crystal violet.

Fig. 5. Chainia barodensis. Sclerotial hyphae with many adjacent sclerotial cells showing enlargement, rounding up and active multiplication in different planes, thereby giving rise to several miniature sclerotia (arrows); GAA, tannic acid-crystal violet.

Fig. 6. Chainia barodensis. Mycelial growth showing many stages in the development of sclerotial hyphae and sclerotia. Note the abnormally large size of the sclerotial units, normal hyphae in background, OMA, phase contrast.

Fig. 7. Chainia 33br. Irregular masses of sclerotial cells; GAA, phase contrast. 

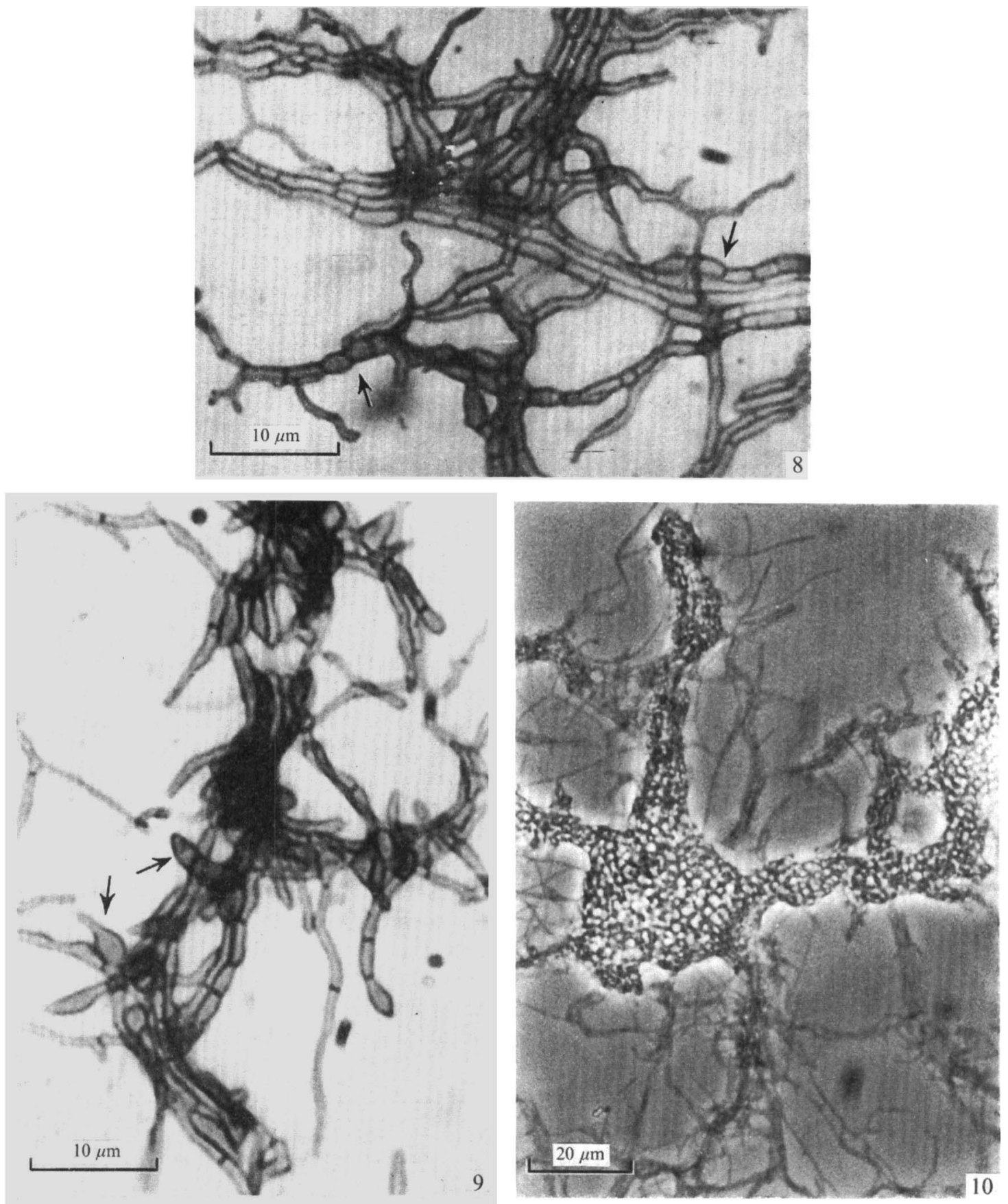

Fig. 8. Chainia 33br. Multihyphal strands with some intercalary sclerotial units (arrows); GAA, tannic acid-crystal violet.

Fig. 9. Chainia ochracea. Advancing mycelial growth showing strands of septate hyphae. Note the origin of spindle-shaped sclerotial buds on these strands (arrows); GAA, tannic acid-crystal violet.

Fig. 10. Chainia ochracea. Intense sclerotial activity along the hyphal strands; GAA, phase contrast. 

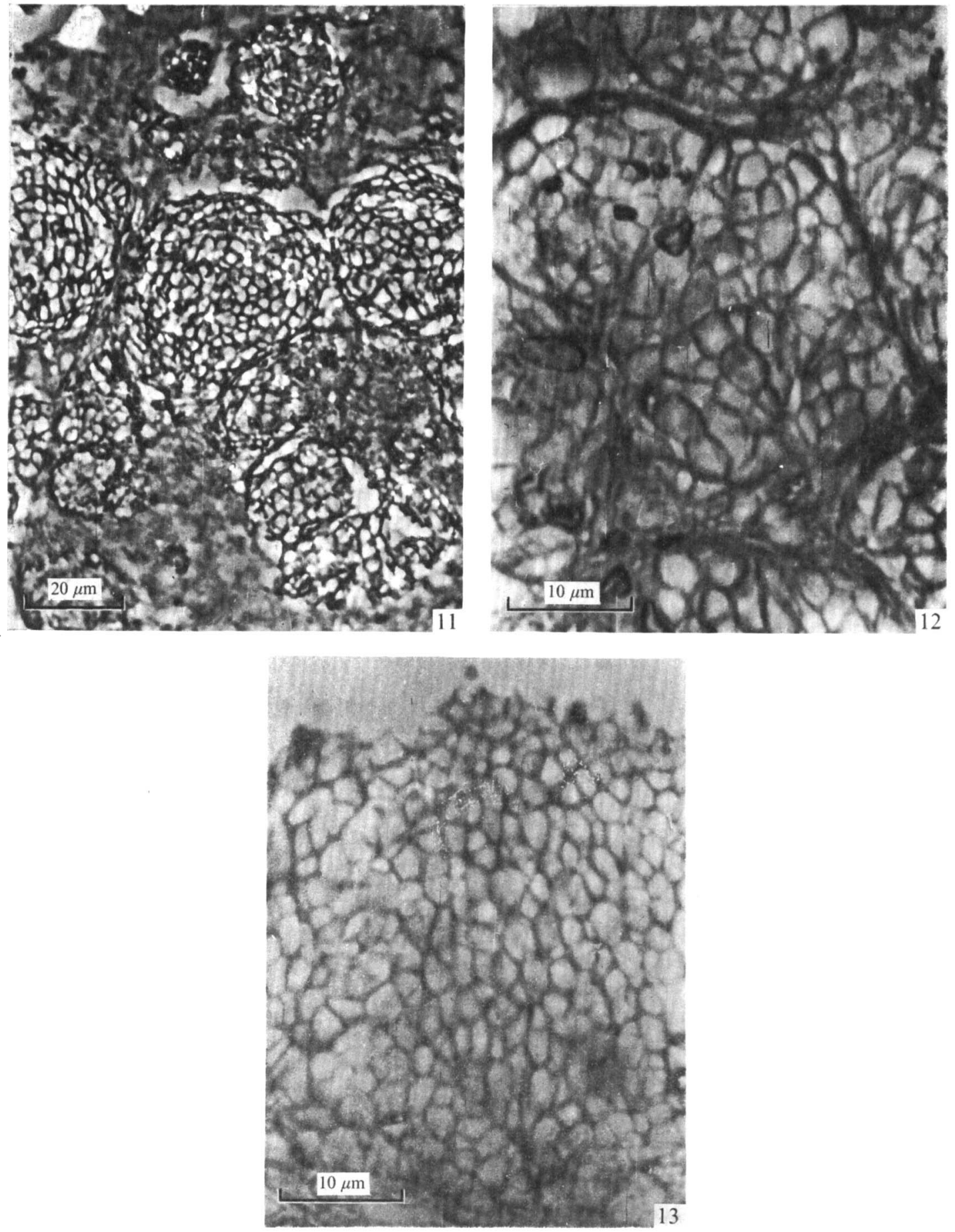

Fig. I I. Chainia barodensis. A longitudinal tangential microtome section of a 15-day-old growth on GAA showing many sclerotia in cross-section; phase contrast.

Fig. I 2. Chainia barodensis. As in Fig. I I, with many adjacent sclerotia in cross-section and showing their internal structure; safranin.

Fig. 13. Chainia 33br. A longitudinal tangential microtome section of 13-day-old growth on YEA showing the structure of stromal sclerotial growth; phase contrast. 

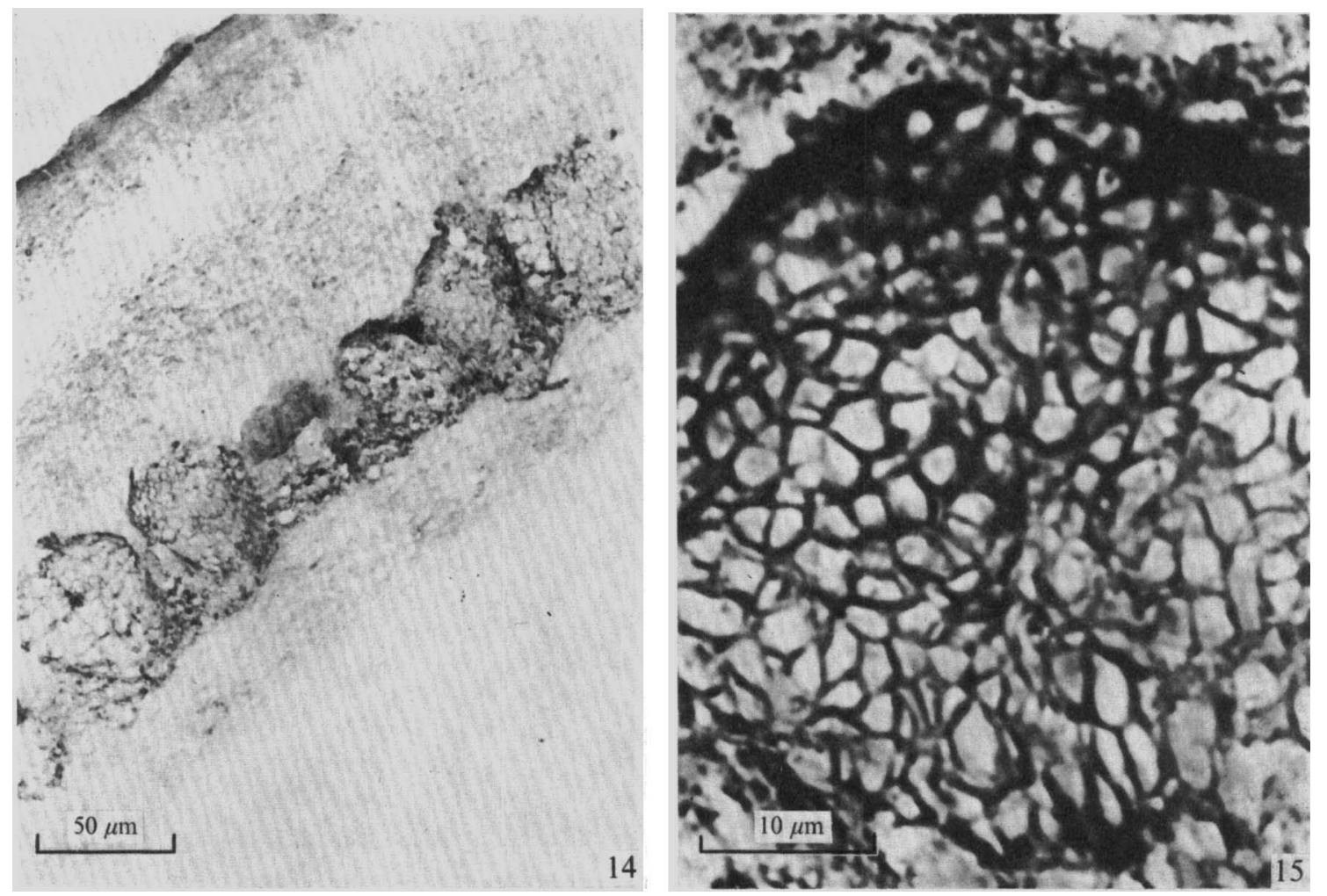

Fig. 14. Chainia ochracea. A longitudinal tangential microtome section of 21 -day-old growth on GAA, showing a row of sclerotia submerged in the mycelial mat; safranin.

Fig. 15. Chainia ochracea. As in Fig. I4, but magnified and showing the internal structure of the sclerotia; safranin.

\section{Sclerotium development in Chainia ochracea}

The development of the initial stages of sclerotial growth in $C$. ochracea appeared to be different from the normal pattern exhibited by the other cultures described above but was similar to Chainia $33 \mathrm{br}$ in many respects. Its characteristic morphological feature was the formation of multihyphal strands along the main hyphae of the advancing mycelium. The hyphae in these composite filaments increased in size and divided extensively by laying down additional septa. The sclerotial buds generally arose laterally on these hyphae and formed sclerotial filaments by further multiplication (Fig. 9). Sclerotial buds were also observed to arise laterally on the side branches of the advancing hyphae. In C. ochracea the sclerotial activity appeared to be very profuse along the multihyphal strands (Fig. Io). Sclerotiumforming initials with septation in different planes were not observed. Discrete sclerotia were also not found in the coverslip cultures.

\section{Anatomy of the sclerotium}

The internal structure of the sclerotia formed by various cultures was studied in thin longitudinal-tangential sections of growths bearing sclerotia obtained from the agar-plate cultures. In Chainia barodensis grown on GAA, discrete sclerotia were formed profusely in the mycelial mat throughout its thickness and were surrounded by loose sclerotial filaments (Fig. I I). Sometimes, many discrete sclerotial bodies were formed very close to each other 

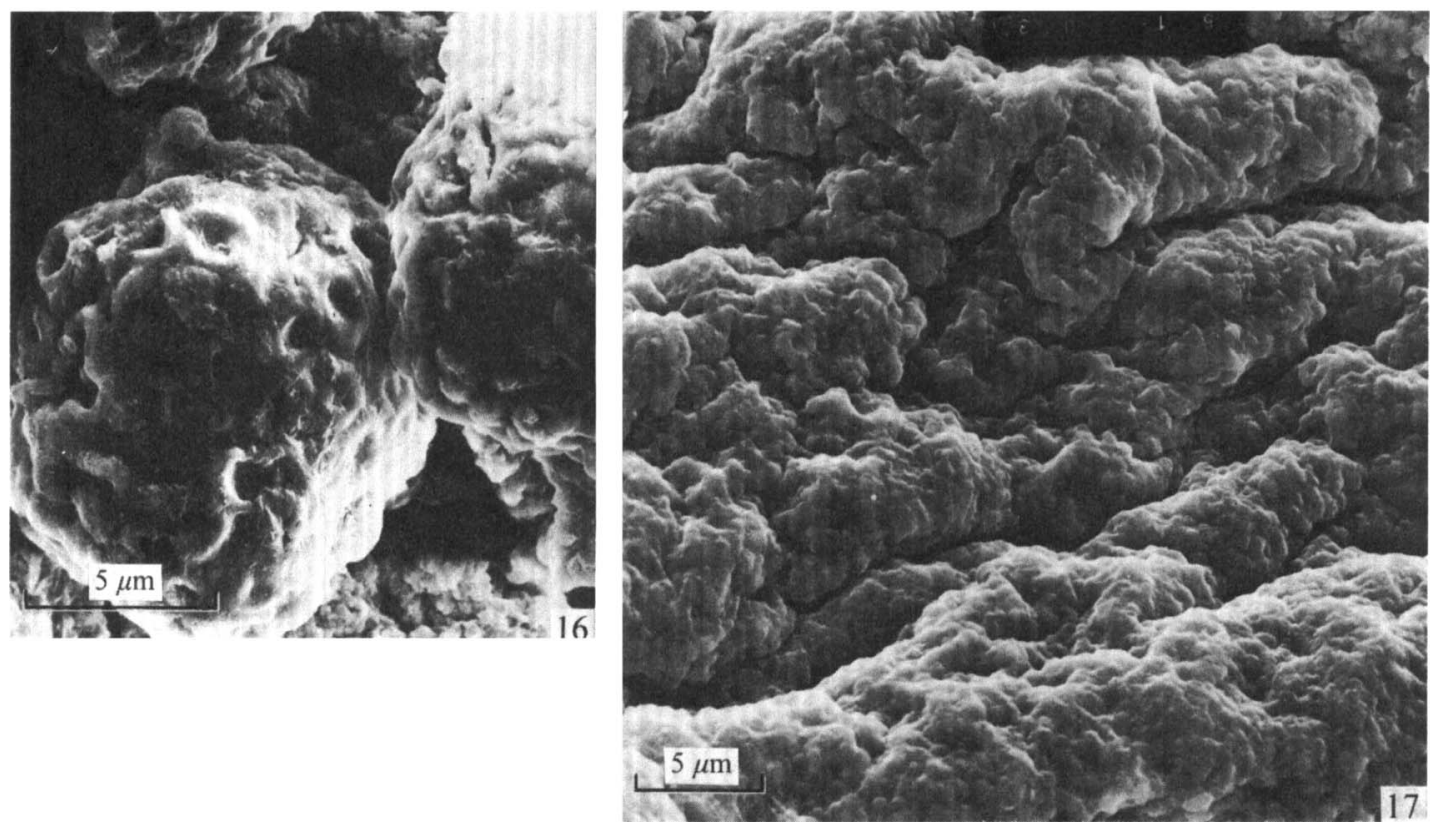

Fig. 16. Chainia barodensis. Scanning electron micrograph of a sclerotium showing its surface architecture; GAA.

Fig. 17. Chainia ochracea. Scanning electron microscope picture of surface growth on YDA, showing sclerotial crust.

(Fig. 12). In Chainia 33br the sclerotia were formed in the uppermost layers of the growth and appeared to be anchored to it. Sometimes discrete sclerotia were not delimited and the cultures formed continuous stretches of compact sclerotial growth resembling a stroma (Fig. 13). Chainia poonensis and $C$. ochracea revealed more or less similar structure as Chainia 33 br. In C. ochracea, the cultures sometimes formed a soft stromal crust of sclerotial hyphae and the sclerotia were submerged in it (Figs. I4 and 15).

Microtome sections of all the sclerotial actinomycetes revealed that the sclerotia were generally discrete multicellular structures of definite configuration. Cross-sections of the sclerotia showed that they had a fine pseudoparenchymatous structure composed of large irregular polygonal sclerotial cells without any spaces between (Figs. 12 and I5). The internal structure of the sclerotia of $C$. barodensis and Chainia 33 br did not suggest any organization of the cells into rings or zones. There was no indication of differentiation into a clear rind, cortex or medulla. In $C$. ochracea, however, the outermost layer of the sclerotial cells showed a dark thickened surface suggesting rind formation.

\section{Scanning electron microscopy of the mature sclerotium}

The external structure of mature sclerotia was difficult to observe in the light microscope. Recourse to s.e.m. revealed some special features of their surface architecture. The sclerotia of Chainia barodensis obtained from YEA cultures presented a rough exterior with a large number of irregularly distributed craters and ridges (Fig. I6). The stromal crust of sclerotial growth exhibited by $C$. ochracea is shown in Fig. I7. 

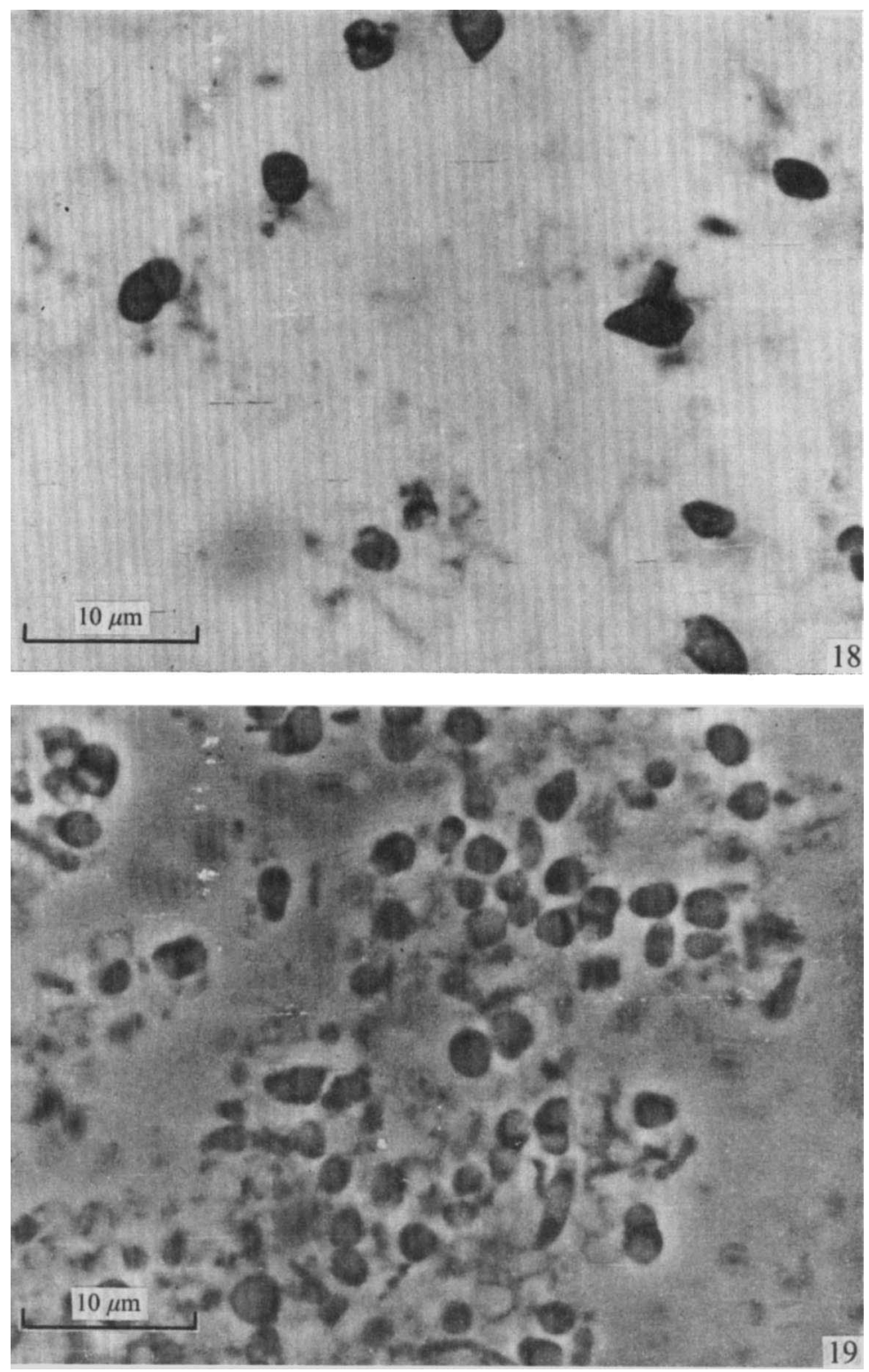

Fig. I8. Chainia barodensis. Sclerotial units obtained from sclerotia crushed between a slide and a coverslip; note the irregular shape of the cells; YEA, tannic acid-crystal violet.

Fig. 19. Chainia 33br. Sclerotial units directly obtained from a slant culture on nutrient agar (Difco) supplemented with $0.4 \%$ gelatin; phase contrast.

\section{Sclerotial units}

When sclerotia of Chainia barodensis, harvested from YEA slants ( 2 days old), were subjected to physical pressure (as between a slide and a coverslip) a large number of predominantly unicellular bodies were released. These detachable sclerotial units could easily be mistaken for fat globules. A fraction of these units was examined microscopically, directly or after staining. The walls of the sclerotial units were prominent in the stained preparations but the internal structure was only faintly visible. Sclerotial units were highly variable in 
shape and size (Fig. I8) and these variations appeared to result from the different physical forces to which the units had been subjected during their growth and expansion within the sclerotium. Sclerotial units of Chainia 33br, obtained directly from Bacto nutrient agar (Difco) supplemented with $0.4 \%$ gelatin, which did not support the formation of discrete sclerotia, are shown in Fig. 19.

\section{Germinability of sclerotial units and sclerotia}

Attempts were made to grow the single sclerotial units of Chainia barodensis freshly isolated from the sclerotia obtained from I5-day-old YEA slant cultures. The sclerotial units were suspended in yeast extract-malt extract broth. Samples ( $\mathrm{I}$ to $2 \mathrm{ml}$ ) of the suspension were placed in test tubes, agitated on a rotary shaker and examined microscopically. Drops of the suspension were also inverted on glass slides and incubated in a moist chamber under suitable conditions. No germination was observed over a period of 3 days. It is not known whether the solitary sclerotial units lose their viability when the parent bodies are disintegrated, or whether they require special conditions for germination.

When intact sclerotia from YEA slants were transferred to fresh medium, visible growth occurred within 2 days. It was not, however, possible to ascertain whether the growth took place by the germination of the sclerotia or simply by an extension of the hyphae surrounding them.

\section{DISCUSSION}

Rapid sclerotization of the mycelium, leading first to the formation of broad closely septate sclerotial hyphae and then to sclerotia which, in cross-section, exhibit a compact pseudoparenchymatous structure, was found to be a characteristic morphological feature of the chainiae.

Streptomyces cultures are known to produce granules which are mere hyphal accretions. It was on the basis of the presence of such granules in two cultures of Streptomyces griseus, including S. griseus ATCCIOI 37, that Gattani (1957) refuted the validity of using sclerotia as a generic criterion. Similar granules in other actinomycetes have also been described by Baldacci et al. (1966). These granules were hard structures of undefined morphology and were formed only on Oxoid nutrient agar. Though these authors stated that a mycelial structure was seen when the granules were treated with hydrochloric acid, they recognized that these bodies were not identical with sclerotia. It was perhaps for this reason that Baldacci \& Locci (1966) accorded to Chainia the status of subgenus and further pointed out that additional knowledge of these structures was necessary.

Our studies of the sclerotium, which included a comparison with one of the strains or Streptomyces griseus studied by Gattani (1957), show that the sclerotia of Chainia are not merely hyphal accretions and that they are different from the granules described by Baldacci et al. (1966) which were produced only on one type of nutrient agar. They also show that the sclerotia of chainiae are distinctive morphological entities. This can be further examined on the basis of the major peculiarities of the cultures studied:

(i) Formation of cross septa during sclerotium development. The ontogeny of sclerotia formed by Chainia species exhibited a series of definite stages. During the early stages of sclerotium development in $C$. barodensis the sclerotial cells divided by both transverse and longitudinal septa. This type of septation was also observed, though less frequently, in C. poonensis and Chainia 33br. However, since these two organisms formed discrete sclerotia whose internal structure was identical with that in C. barodensis, it is presumed that they formed these bodies in a similar way to that observed in the latter species. 
In a thin microtome section of a fully-formed sclerotium, septa could be observed in different planes but it was difficult to be certain that this was true cross-septation. It could be argued that the observed phenomenon was the result of a change in the orientation, within the main axis, of the hyphae which ultimately form the sclerotia. That this was not so and that there was true cross-septation in different planes was clearly demonstrated in the early developmental stages of the sclerotia.

Septation of the hyphae in different planes is unknown in the genus Streptomyces and in actinomycetes outside the genus Dermatophilus (which is placed in a separate family on the basis of this character). Thompson \& Bisset (I957) recommended that a suborder of the Eubacteriales be created to accommodate their new genus Polysepta which formed crosswalls in different planes; Polysepta has been subsequently re-classified as Dermatophilus. The point that is to be emphasized here is that the sclerotial hyphae and sclerotia exhibited a form of cross-septation which is rare in actinomycetes and unknown in Streptomyces. Chainia, however, cannot be included in Dermatophilus because of other characteristics of the two genera.

(ii) Origin. Thirumalachar (1955) suggested that the sclerotia in Chainia were formed by an incurling of hyphal strands accompanied by close septation and enlargement of the resulting units. In the sclerotial actinomycetes studied here alternative modes of development have been recognized. Thus, in $C$. barodensis and $C$. poonensis the sclerotium had an intercalary origin among sclerotial hyphae which distinguished themselves from the thin primary hyphae by prior enlargement. In C. ochracea, the sclerotia originated from lateral buds which developed from hyphae along a stroma of multihyphal origin in which they remained anchored. Chainia 33 br shared the features of both $C$. barodensis and C. ochracea in that sclerotia developed both from intercalary positions as in $C$. barodensis and from lateral buds as in $C$. ochracea. This organism also exhibited the formation of a stroma-like tissue in which the sclerotia were embedded.

The differences in development and the stroma formation naturally raise the possibility of further differentiating the sclerotial actinomycetes on the basis of these characters. However, such a step must await similar studies on other sclerotial species.

(iii) Surface features of sclerotia. The embedded nature of the sclerotia in Chainia ochracea and Chainia $33 \mathrm{br}$ made it difficult to consider the surface configuration of sclerotia. However, in $C$. barodensis where the sclerotia are separate, s.e.m. indicated a distinctive surface architecture; the sclerotial surface had a moon-like surface topography with numerous ridges and craters. In C. ochracea, s.e.m. of the surface growth of a colony grown on an agar medium clearly revealed a stromal crust of sclerotial growth. These features of the sclerotium in $C$. barodensis and the growth of $C$. ochracea point to the need for further study of the surface features of other species of Chainia. It is reasonable to assume, on the basis of these observations, that such a study should add to our understanding of the speciation of sclerotial actinomycetes forming individual sclerotia.

Comparison of actinomycete sclerotia with fungal sclerotia. In fungi, three types of sclerotium development are known (Willetts, 1972): the loose type in which the sclerotia are formed by intercalary septation of irregularly branched hyphae which lack a definite pattern of organization, and the terminal and lateral types in which the sclerotia exhibit a welldefined pattern of development. The sclerotia in the actinomycetes of our study, as represented by Chainia barodensis and C. poonensis, have an intercalary origin in hyphae, but are not of the loose type and possess well-defined developmental stages and structure. The terminal type has not been observed in the actinomycetes studied by us. The lateral type, in which sclerotia are formed by the interweaving of side branches which arise from one or 
more hyphae, seems to have some similarity to the development observed in $C$. ochracea and Chainia 33 br.

Formation of cross septa in different planes, observed in the early stages of development of several sclerotial actinomycetes, has not been described in the development of fungal sclerotia. Furthermore, the sclerotia of some cultures, e.g. Chainia barodensis, could be disorganized by applying mild physical pressure, yielding individual sclerotial units. This kind of disintegration does not seem to have been described in the case of fungal sclerotia.

Thirumalachar (1955) reported that the sclerotia of Chainia antibiotica were easily separable from the cultures and germinated on transfer to fresh media. Such growth from sclerotia has been obtained in these studies also, but this does not necessarily mean that the sclerotia have germinated. Sclerotia in Chainia are invariably covered with actively growing hyphae, and transfer of sclerotia, regardless of the care taken, does not preclude growth from these hyphae. Germination of sclerotia in fungi generally involves growth from the medullar region underneath the rind and it takes place even after the sclerotia have been exposed to extreme environmental conditions. In the absence of a conclusive demonstration of the resistant nature of the actinomycete sclerotia and their germination it is as yet difficult to assign to these structures a functional role similar to that in the fungi.

We thank Dr Y. Okami and JEOL Ltd, Tokyo, Japan, for the s.e.m. pictures, Dr C. H. Pathak for assistance with microtomes, and Mr C. K. Darji for help in the preparation of the coverslip cultures.

\section{REFERENCES}

BALDACCI, E. \& LocCI, R. (1966). A tentative arrangement of the genera of Actinomycetales. Giornale di Microbiologia 14, I3I-I 39.

BaldacCi, E., LocCI, R. \& LocCI, J. R. (1966). Production of 'granules' by Actinomycetales. Giornale di Microbiologia 14, 173-I84.

Butler, G. M. (1966). Vegetative structures. In The Fungi, vol. 2, pp. 83-1 12. Edited by G. C. Ainsworth and A. S. Sussman. New York and London: Academic Press.

GatTani, M. L. (1957). Production of sclerotic granules by Streptomyces sp. Nature, London 180 , I $293-1294$.

Kalakutskil, L. V. \& Krassilnikov, N. A. (1960). The formation of sclerotia by actinomyces and the systematic position of the genus Chainia. Trudy instituta mikrobiologii. Akademiya nauk SSSR 8, 45-55 (cited from Biological Abstracts 37, No. 23153, 1962).

KLieneberger-Nobel, E. (I947). The life cycle of sporing Actinomyces as revealed by their structure and septation. Journal of General Microbiology 1, 22-32.

Kuznetsov, V. D. (1962). A new species of the genus Chainia. Microbiology, USSR 31, 435-439.

Nishimura, H. \& TAWARA, K. (1957). A method for microscopical observation of Streptomyces using agar cylinder culture. Journal of Antibiotics A ro, 82.

Purvis, M. J., Collier, D. C. \& Walls, D. (1964). Laboratory Techniques in Botany. London: Butterworths.

Shirling, E. B. \& GotTlieb, D. (1966). Methods for characterisation of Streptomyces species. International Journal of Systematic Bacteriology 16, 31 3-340.

Shirling, E. B. \& Gottlieb, D. (1972). Cooperative description of type strains of Streptomyces. V. Additional descriptions. International Journal of Systematic Bacteriology 22, 265-394.

Thirumalachar, M. J. (1955). Chainia, a new genus of the Actinomycetales. Nature, London 176, 934-935.

Thirumalachar, M. J., Rahalkar, P. W., Deshmukh, P. V. \& Sukapure, R. S. (I965). Production of aburamycin by Chainia minutisclerotica, a new species of actinomycetes. Hindustan Antibiotics Bulletin 8 , 6-9.

Thirumalachar, M. J. \& Sukapure, R. S. (1964). Studies on species of the genus Chainia from India. Hindustan Antibiotics Bulletin 6, I57-166.

Thirumalachar, M. J., Sukapure, R. S., Rahalkar, P. W. \& Gopalkrishnan, K. S. (1966). Studies on species of the genus Chainia from India. II. Hindustan Antibiotics Bulletin 9, 10-1 5.

Thompson, R. E. M. \& Bisset, K. A. (1957). Polysepta: a new genus and suborder of bacteria. Nature, London 179, 590-59I. 
TUlasNe, R. \& VendRely, R. (1947). Demonstration of bacterial nuclei with ribonuclease. Nature, London I60, $225-226$.

Waksman, S. A. (196I). Classification, identification and descriptions of genera and species. In The Actinomycetes, vol. 2, p. 332. Baltimore: Williams and Wilkins.

WiLLETTS, H. J. (1971). The survival of fungal sclerotia under adverse environmental conditions. Biological Reviews 46, 387-407.

WILLETTS, H. J. (1972). The morphogenesis and possible evolutionary origins of fungal sclerotia. Biological Reviews 47, 515-536.

Williams, S. T. \& DAvies, F. L. (1967). Use of a scanning electron microscope for the examination of actinomycetes. Journal of General Microbiology 48, 171-177. 